\title{
Expression of a ScFv-E2T fusion protein in CHO-K1 cells and alfalfa transgenic plants for the selective targeting to antigen presenting cells
}

Agustín Ostachuk $^{1 *}$, Sebastián Chiavenna ${ }^{1}$, Cristina Gómez $^{2}$, Andrea Pecora ${ }^{1}$, Mariano Pérez-Filgueira ${ }^{1}$, José Escribano $^{3}$, Fernando Ardila ${ }^{2}$, María Dus Santos ${ }^{1}$, Andrés Wigdorovitz ${ }^{1}$

${ }^{1}$ Instituto de Virología, Instituto Nacional de Tecnología Agropecuaria (INTA), Buenos Aires, Argentina; ${ }^{2}$ Instituto de Genética, Instituto Nacional de Tecnología

Agropecuaria (INTA), Buenos Aires, Argentina; ${ }^{3}$ Departamento de Biotecnología, Instituto Nacional de Investigaciones Agropecuarias (INIA), and Algenex, Madrid, Spain

*Corresponding author. E-mail: aostachuk@cnia.inta.gov.ar

INTRODUCTION

Bovine viral diarrhea virus (BVDV) is a pestivirus of the Flaviviridae family. It is an important cause of mortatility, morbidity and economic loss of cattle with a worldwide distribution. Vaccination is an important component of BVDV prevention an control programs. Subunit vaccines provide the opportunity to develop s.
protective immune response to a cost affordable for veterinary applications.

One alternative to solve this problem is to increase vaccine immunogenicity. A central event in the development of adaptive immune response is the activation of $\mathrm{CD}^{+} \mathrm{T}$ cells by antigen presenting cells (APCs). APCs present antigenic peptides to $\mathrm{CD}^{+} \mathrm{T}$ cells on MHC class II molecules. In consequence, a way to obtain a more intense specific immune response is to increase the
number of MHC-peptide complexes on the surface of APCs. This would be possible by targeting antigens to APCs, fusing them to number of MHC-peptide complexes on the surface of APCs. This would be possible by targeting antigens to APCs, fusing them to specific antibodies against APCs' surface markers. In this work, we report the development of a fusion protein between a single chain antibody fragment (ScFv), recognizing a conserved region of MHC class II molecules, and a truncated form of the E2 glicoprotein
(E2T) from BVDV, without its transmembrane domain. E2 is the most immunogenic protein of the virus and therefore is a good
candidate for a subunit vaccine.

Another alternative is the use of transgenic plants as vectors for the expression of viral and bacterial antigens. Transgenic plants have been increasingly tested as an alternative methodology for the production of experimental vaccines, based on their possibility of oral adristration. Feed fodder crops such as alfala are the best option for the development of animal vaccibes ung transgenic plants. However, a major drawback of using transgenic plants as protein factories is the low expression level of the recombinant antigens. The use of strong promoters for conducting transgene expression may permit to overcome this problem. Previous results obtained by our group employing transient expression systems in alfalfa (Medicago sativa), showed that Cassava Vein Mosaic Virus (CsVMV) promoter presents higher transcriptional activity than the standard 3SS promor. Here we report the expressed transiently and stably in CHO-K1 cells and purified from cell culture supernatants as a convenient source of purified protein for functional studies.

RESULTS

Transient expression of ScFv-E2T and E2T in CHO-K1 cells. CHO-K1 cell monolayers were transfected with differen plasmid concentrations. After 24 hs., cell lysates were evaluated by SDS-PAGE and Western blot for the presence of recombinan plasmid concentrations. After $24 \mathrm{hs} .$, ceell lysates were evaluated by SDS-PAGE and Western blot for the presence
proteins. Incubation with a monoclonal antibody specific for E2 revealed the presence of the desired proteins (Figure 1).

Stable cell line generation. Transfected cell monolayers were subcultured in the presence of $700 \mu \mathrm{g} / \mathrm{ml}$ Geneticin (Invitrogen) for 21 days. Transfection with a plasmid containing the LACZ coding region was used for monitoring the progress of the selection process. After 14 days, groups of cells resistant to the antibiotics (blue-coloured) were evident, as revealed by betagalactosidase activity using X-Gal as substrate (Figure 2). At day 14 and 21, cells were subcultured by limiting dilution in 96-well microplates in order to obtain stable and amplified recombinant clones. Positive clones
constructs (red-circled, Figure 3). These clones were amplified and stored in liquid nitrogen.

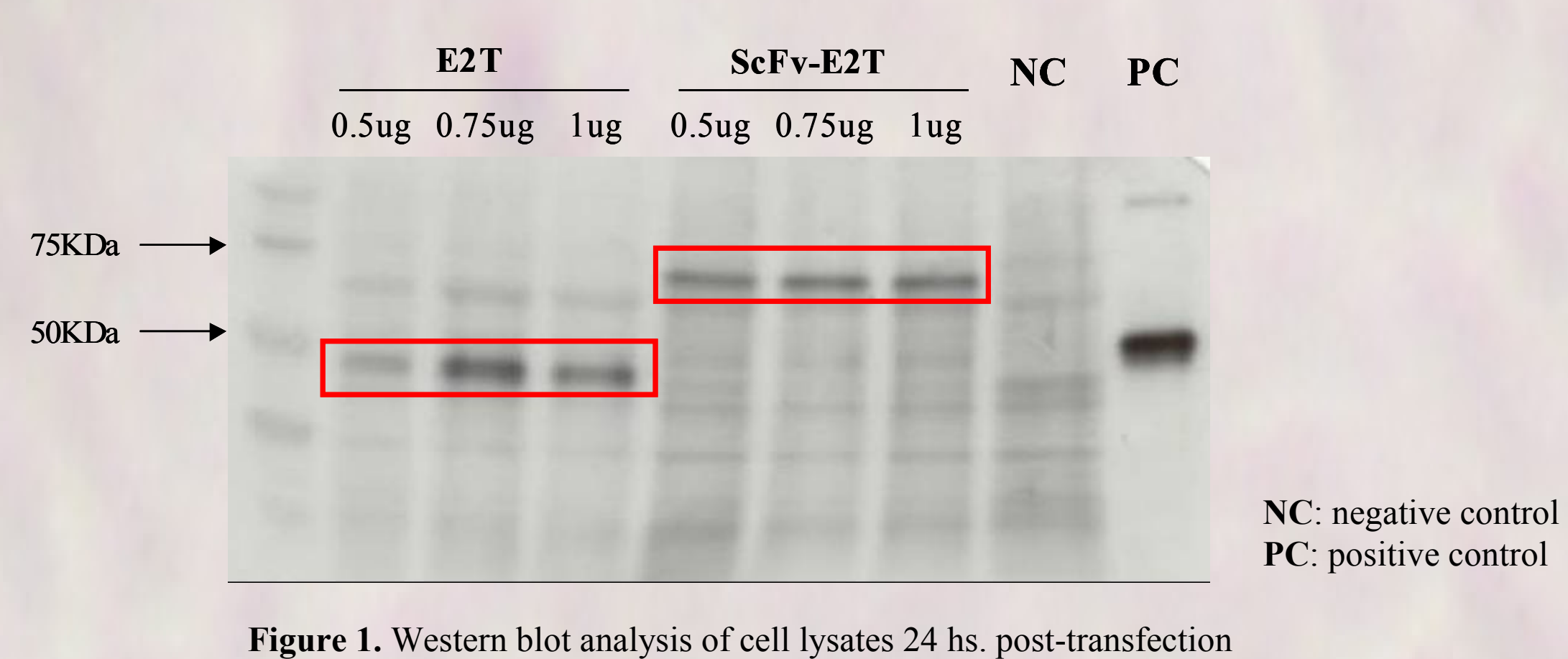

Day 1

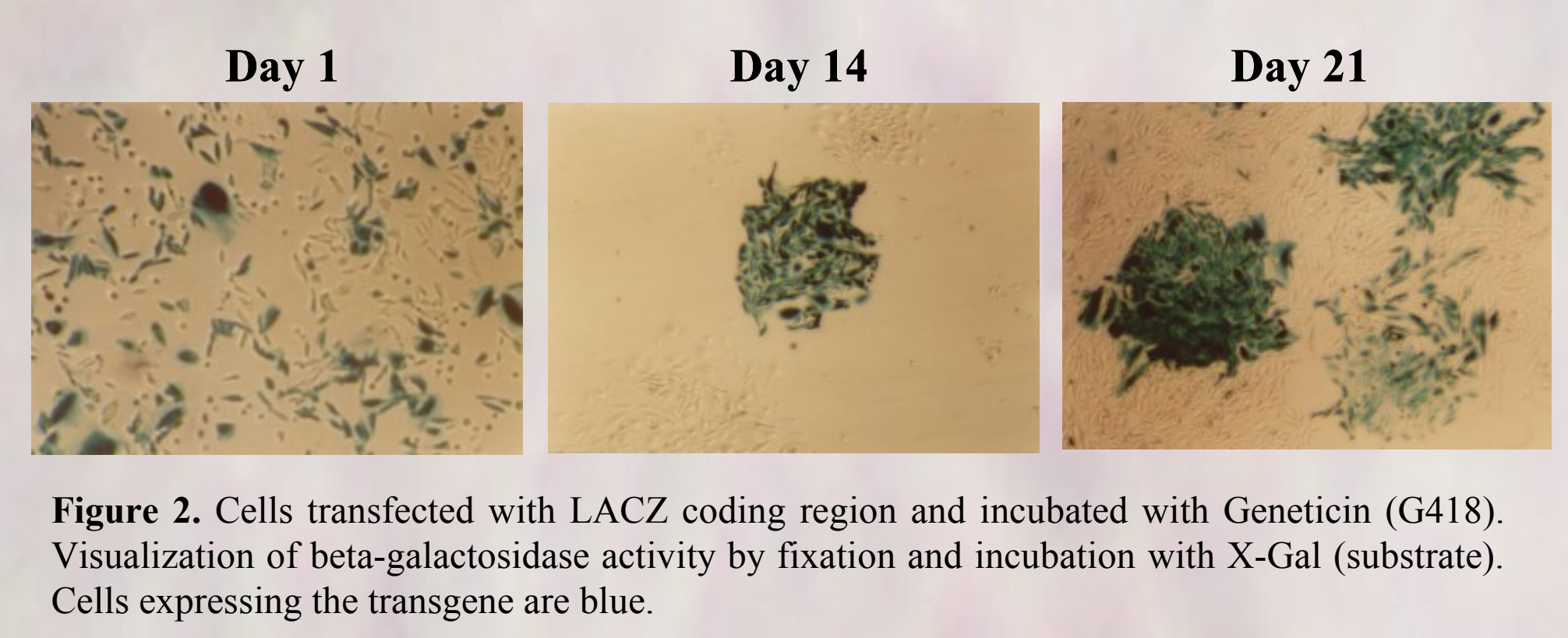

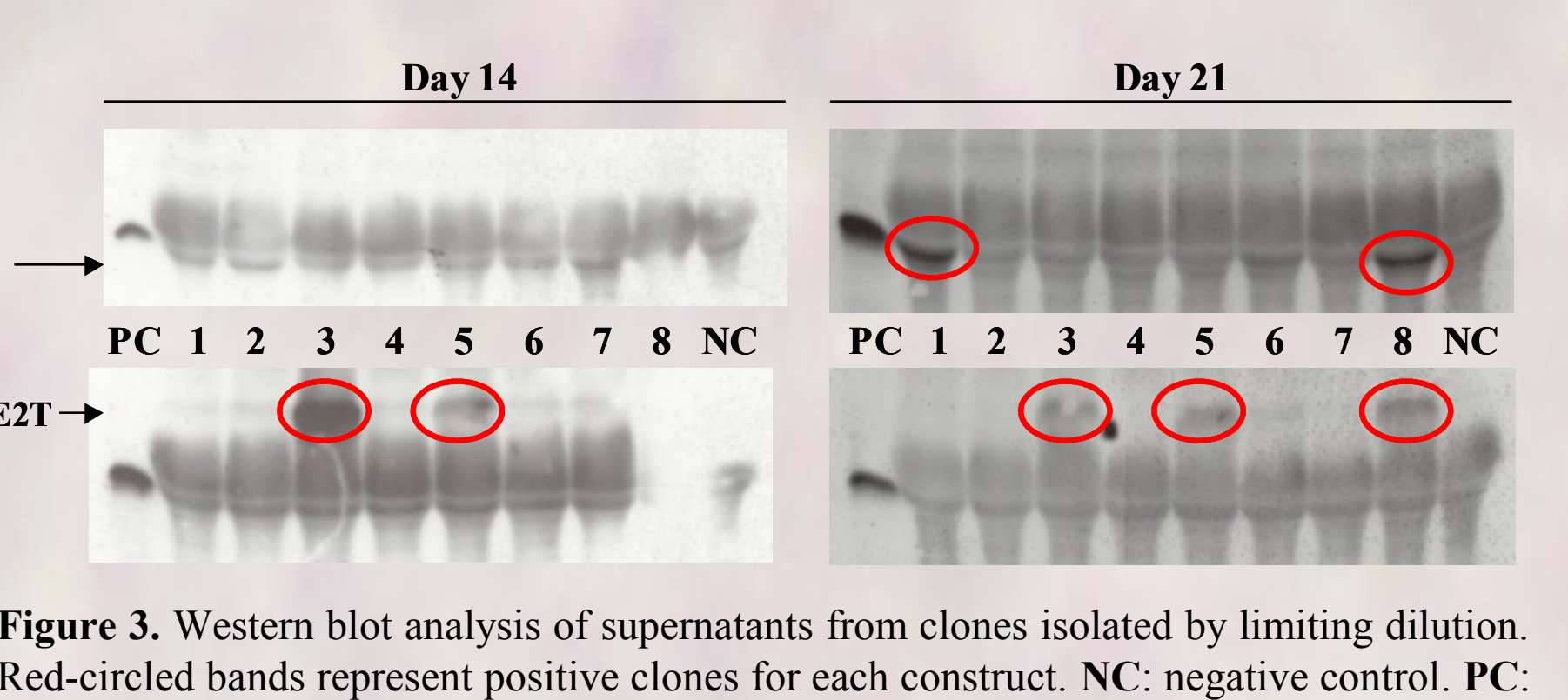

Characterization of recombinant ScFv-E2T and E2T expression in stable cell lines. Recombinant protein in cell culture supernatants was measured by ELISA. Clones with highest expression levels were chosen to carry out future experiments. Results indicated that product accumulates exponentially during the first 7-8 days and then reaches a plateau (Figure 4). Furthermore,
addition of DMSO $(2 \%)$ and sodium butyrate $(2 \mathrm{mM})$ to cell culture medium notably increased expression levels of recombinant addition of DMSO $(2 \%)$ and sodium butyrate $(2 \mathrm{mM})$ to cell culture medium notably increased expression levels of recombinant proteins (4-fold increase). Product accumulation kinetics was similar using flasks and roller bottles, with even better yields in the atter case (data not shown).

Recombinant protein purification by IMAC. ScFv-E2T and E2T were purified by IMAC. Most proteins of cell culture supernatant remained unbound to the resin whereas recombinant proteins could be obtained in eluates with high purity (Figure 5). In the case of ScFv-E2T, some protein degradation is evident in spite of having used protease inhibitors. Proteins 'identity was
confirmed by Western blot. Recombinant proteins could be obtained with a purity of approximately $80 \%$ and an average yield of the whole process of $40 \%$, being capable of purifying $134 \mu \mathrm{g}$ of recombinant protein from $1 \mathrm{~L}$ of cell culture supernatant (with an initial concentration of $334 \mu \mathrm{g} / \mathrm{L}$ ) (data not shown).

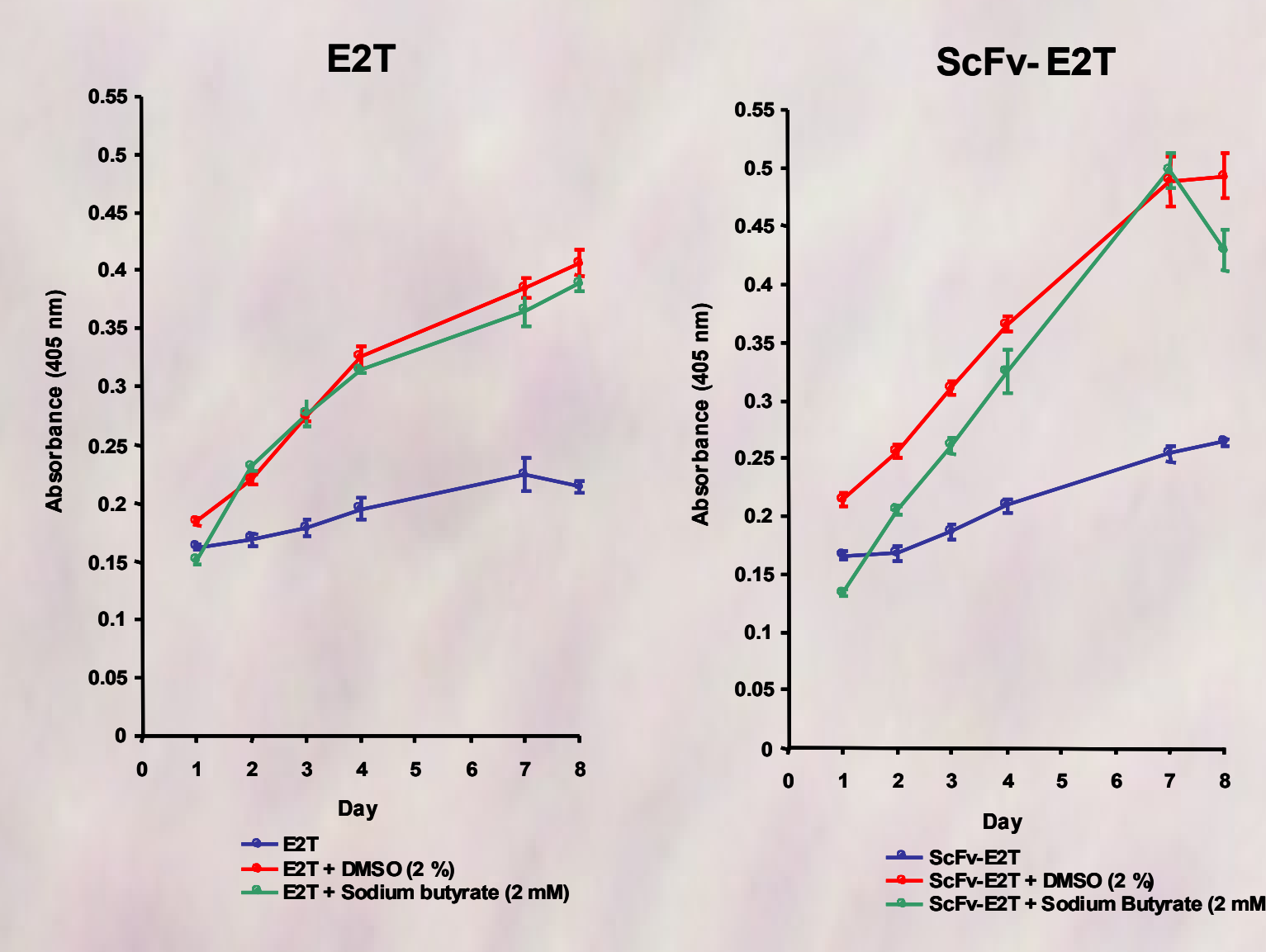

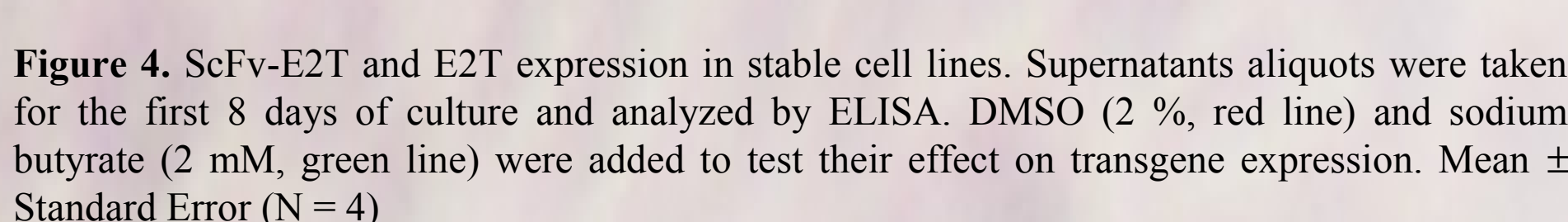

Binding of ScFv-E2T and E2T to the surface of PBMCs. In order to test the ability of recombinant proteins to bind to the surface of APCs as the first step of antigen presentation, PBMCs from different species (bovine, pig, horse, goat, sheep, guinea pig and mice) were purified from heparinized blood with Ficoll-Paque Plus (Amersham), and used for surface labeling by flow cytometry. ScFv-E2T was capable of binding to the surface of PBMCs of all species except for mice, most presumably due to the E2T) bound more intensively to PBMCs than the untargeted protein (E2T), demonstrating that targeting proteins to MHC class II
I molecules consist of an efficient strategy to augment antigen delivery to antigen presenting cells.

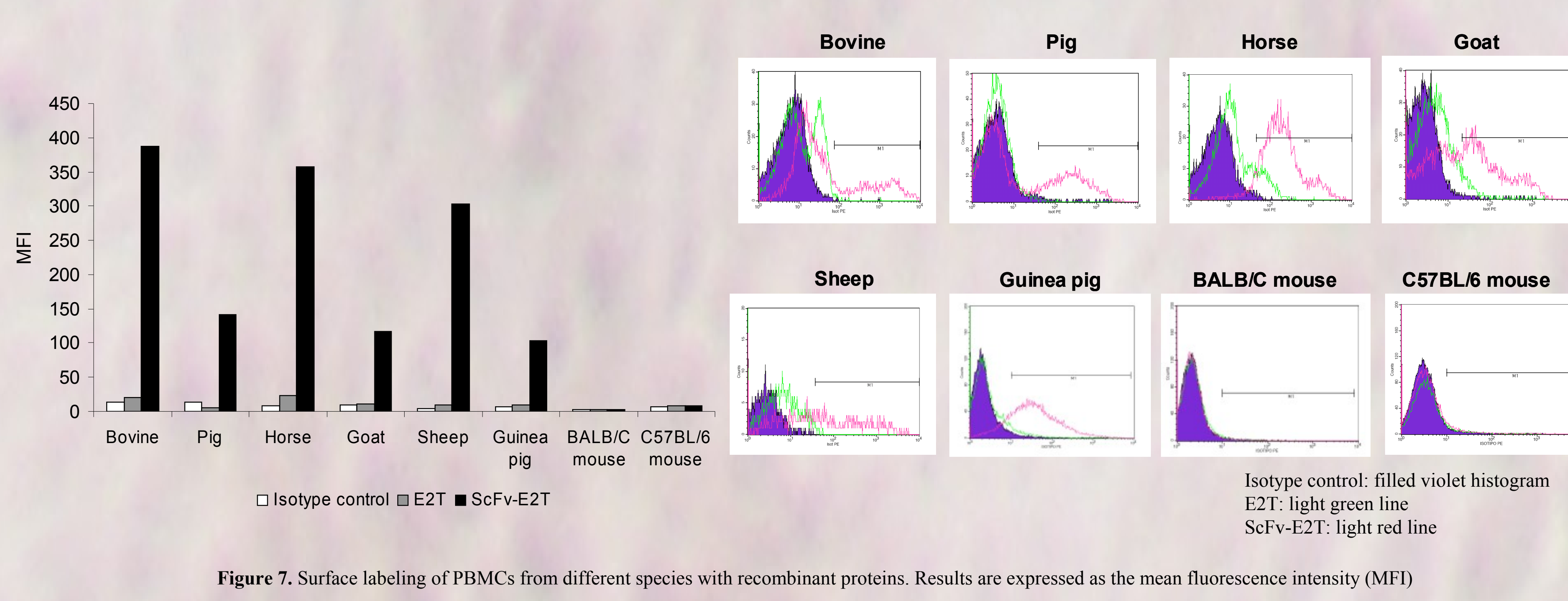

In vitro $\mathrm{T}$ cell proliferation by bovine APCs. To investigate if the improved antigen delivery to the surface of APCs mediated by targeting to MHC class II molecules resulted in a concomitant increase on the number of MHC-peptide complexes on APCs (mainly macrophages). ScFv-E2T generated a higher T cell proliferation measured by ${ }^{3}[\mathrm{H}]$ thymidine incorporation than E2T at all concentrations (Figure 8). Moreover, E2T seemed to be not capable of inducing a T cell proliferation of the magnitude and same T cell proliferation induced by E2T. In consequence, ScFv-E2T seems to be capable of inducing T cell proliferation at even lower concentrations, whereas E2T could not.

Characterization of recombinant ScFv-E2T and E2T expression in alfalfa transgenic plants. Alfalfa transgenic plants were analyzed for the expression of E2T and ScFv-E2T fusion protein by ELISA. Expression levels in plants were very similar to
those obtained in CHO-K1 cells (Figure 9), extracting approximately $1 \mu \mathrm{g}$ of protein per gram of leaf (data not shown).
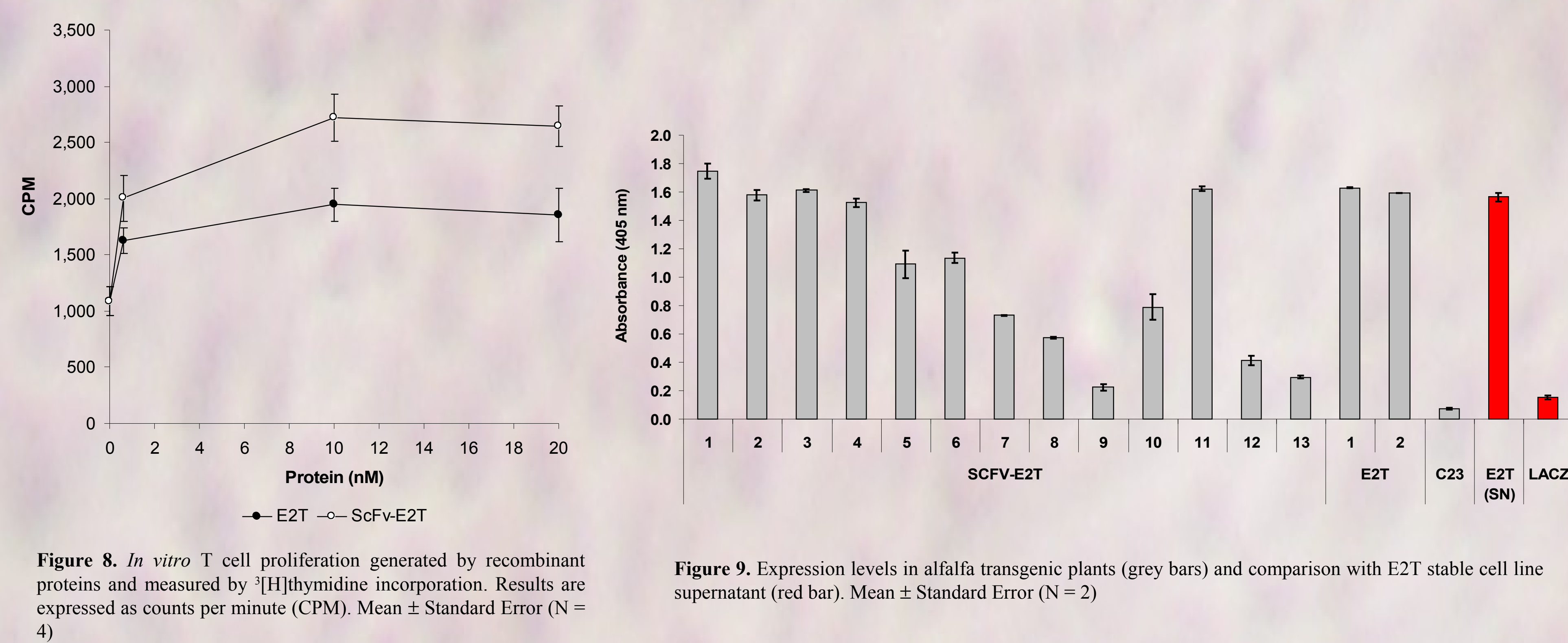

\section{CONCLUSIONS}

- Binding of recombinant proteins to the surface of PBMCs revealed that ScFv-E2T (targeted antigen) bound more intensively than E2T (untargeted antigen), demonstrating that targeting proteins to MHC class II molecules consist of a

efficient strategy to increase antigen delivery to APCs.
- In an in vitro bovine model of $T$ cell proliferation, ScFv-E2T generated a more potent specific $T$ cell response than E2T. This means that antigen delivery to APCs through targeting to MHC class II molecules would result in a vaccine with higher immunogenicity than the conventional non-targeted vaccinc.

- Expression levels in affalfa transgenic plants were very similar to mammalian cells and estimated in $1 \mu \mathrm{g}$ of recombinant protein per gram of leaf, providing evidence for the viability of using transgenic plants as system for subunit vaccine

MATERIALS AND METHODS

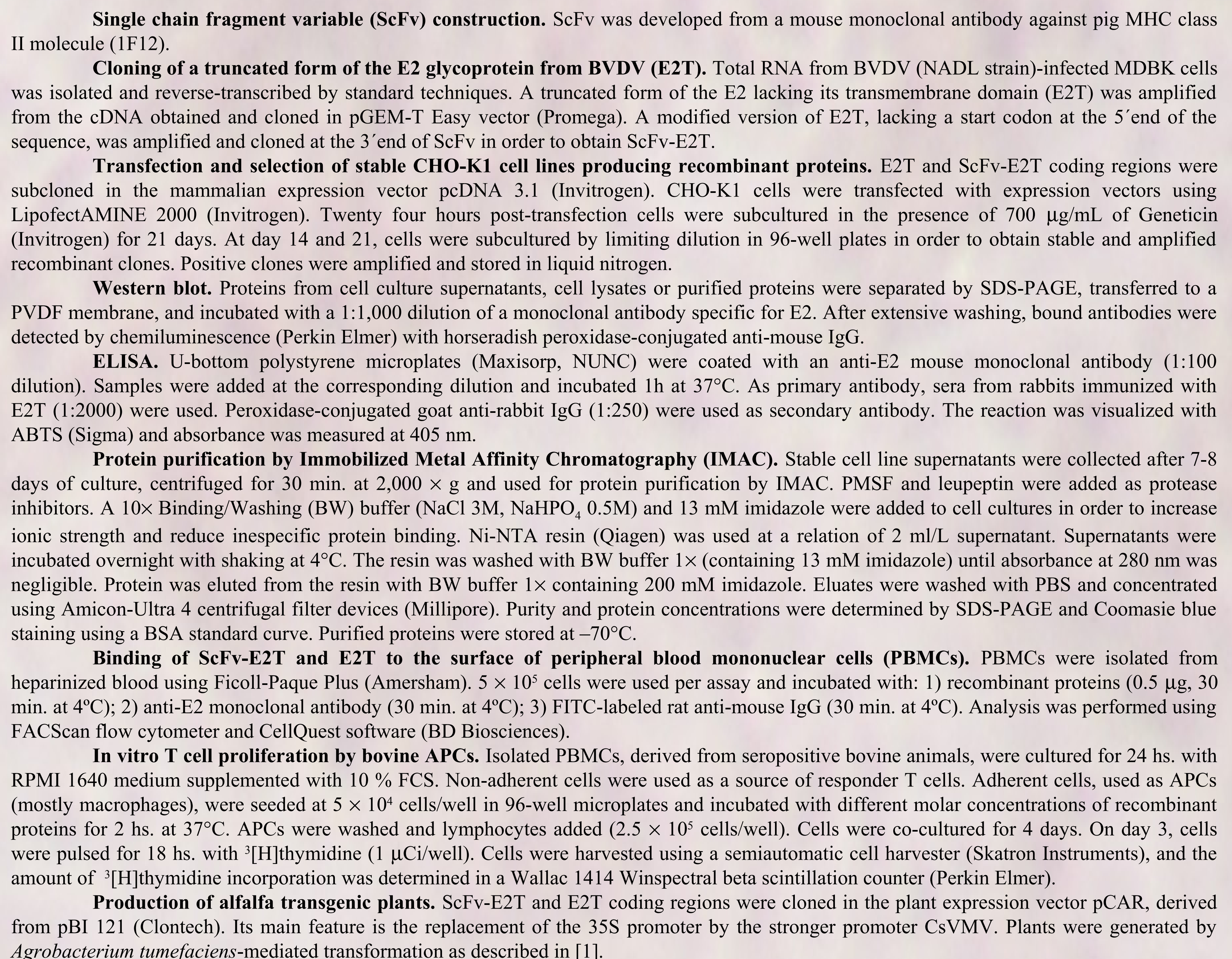

